\title{
Gravitational Redshift and Dark Matter: Application to SO-2's Case
}

\author{
Stéphane Le Corre \\ Ecole Polytechnique Fédérale de Lausanne, Lausanne, Switzerland \\ Email: stephane.lecorre@epfl.ch
}

How to cite this paper: Le Corre, S. (2018) Gravitational Redshift and Dark Matter: Application to SO-2's Case. Open Access Library Journal, 5: e4607.

https://doi.org/10.4236/oalib.1104607

Received: April 19, 2018

Accepted: May 15, 2018

Published: May 18, 2018

Copyright $\odot 2018$ by author and Open Access Library Inc.

This work is licensed under the Creative Commons Attribution International License (CC BY 4.0).

http://creativecommons.org/licenses/by/4.0/

(c) (i) Open Access
Abstract
The star SO-2 at the galactic center will be soon at its closest distance to the
supermassive black hole (SMBH). It will allow measuring relativistic effects.
In [1] the dark matter is explained by the second component (gravitic field) of
the general relativity generated by the clusters. In this theoretical frame, the
gravitic field of the galaxies cannot explain the dark matter at their ends. But despite this, it seems possible that this gravitic field is in general underestimated. In the current paper, we study the component of the SO-2's gravitational redshift due to the gravitic field of the galactic center $\left(z_{H}\right)$ compared to the expected gravitational redshift due to the gravity field $\left(z_{G} \sim 3 \times 10^{-4}\right)$. The value of the gravitic field of SMBH is not known but depending on its value, four cases (in agreement with general relativity) can be obtained. If the discrepancy measured on the gravitational redshift of SO-2 is $z_{H} \ll 10^{-5}$, it will mean that the gravitic field at the center of the Galaxy is too weak to be measured and, as expected, that the gravity field dominates. If a discrepancy is measured of around $z_{H} \sim 10^{-5}$, the gravitic field at the Galaxy center will be greater than expected but always inferior to the effect of the gravity field. With a measure of around $z_{H} \sim 10^{-4}$, this discrepancy could always be explained in agreement with general relativity. It will mean that the effect of the gravitic field at the Galaxy center is greater than expected and can even be of the same order of magnitude than the effect of the gravity field. Furthermore the calculation of the mass could have to be revised. If a discrepancy is measured of around $z_{H} \sim 10^{-3}$ this discrepancy could always be explained in agreement with general relativity. It will mean that the effect of the gravitic field at the Galaxy center is greater than expected and even greater than the effect of the gravity field. The calculation of the mass will have to be revised. In the three previous cases, this discrepancy will be a measure of the gravitic field of $\mathrm{SMBH}$ and would be an important clue that would indirectly corroborate the explanation of the dark matter as the effect of the term of gravitic field. And to 
end, if the discrepancy is larger, it will be more difficult to explain it in the frame of the general relativity (even in the frame of the explanation of dark matter as the effect of the term of gravitic field).

\section{Subject Areas}

Modern Physics, Theoretical Physics

\section{Keywords}

Galaxies, Fundamental Parameters, Galaxies, General, Galaxies, Kinematics and Dynamics, Dark Matter

\section{Introduction}

General relativity implies the existence of two gravitational components. In addition to the gravity field, there is a gravitic field just like the magnetic field in electromagnetism. These both components give what is called the gravitomagnetism, obtained from the linearization of the general relativity. This new gravitic field can be measured by its effect, known as Lense-Thirring effect. Several experiments have validated this effect for the Earth gravitic field, NASA's LAGEOS satellites or Gravity Probe B [2]. In [1], a solution is proposed to explain the dark matter, compliant with general relativity and without exotic matter. This explanation leads to the assumption that we are embedded in a relatively uniform gravitic field generated by larger structures than galaxies (likely the clusters). More generally, this solution implies that large structures generate greater gravitic field (and then Lense-Thirring effect) than expected. In particular the center of galaxies could be a first example of such a phenomenon. Even if the gravitic field of the galaxies' center can't explain the dark matter at the ends of the galaxies [1], it could be locally an important component of the dynamic of the object near the center (perhaps of the same order of the gravity field). The goal of our study is to predict what could be the effect of the gravitic field at the center of our galaxy $\left(\operatorname{Sgr} A^{*}\right)$ on the measure of the gravitational redshift of the star SO-2 which will be soon very close to SgrA*. That will allow testing the general relativity in extreme conditions. We will see that unexpected discrepancies are possible due to this gravitic field. Some of these discrepancies could corroborate the previous mentioned solution of dark matter (and then by the same time could be in agreement with general relativity). Let's first demonstrate what general relativity gives for the expressions of the gravitic field, which will allow giving us an expression for the gravitational redshift.

\section{Dark matter Explained by General Relativity}

\subsection{From General Relativity to Linearized General Relativity}

From general relativity, one deduces the linearized general relativity in the ap- 
proximation of a quasi-flat Minkowski space $\left(g^{\mu \nu}=\eta^{\mu \nu}+h^{\mu \nu} ;\left|h^{\mu \nu}\right| \ll 1\right)$. With the following Lorentz gauge, it gives the following field equations as in [3] (with $\left.\square=\frac{1}{c^{2}} \frac{\partial^{2}}{\partial t^{2}}-\Delta\right)$ :

$$
\partial_{\mu} \bar{h}^{\mu v}=0 ; \square \bar{h}^{\mu v}=-2 \frac{8 \pi G}{c^{4}} T^{\mu v}
$$

With:

$$
\begin{gathered}
\bar{h}^{\mu \nu}=h^{\mu \nu}-\frac{1}{2} \eta^{\mu \nu} h ; h \equiv h_{\sigma}^{\sigma} ; h_{v}^{\mu}=\eta^{\mu \sigma} h_{\sigma v} ; \\
\bar{h}=-h
\end{gathered}
$$

The general solution of these equations is:

$$
\bar{h}^{\mu v}(c t, \boldsymbol{x})=-\frac{4 G}{c^{4}} \int \frac{T^{\mu v}(c t-|\boldsymbol{x}-\boldsymbol{y}|, \boldsymbol{y})}{|\boldsymbol{x}-\boldsymbol{y}|} \mathrm{d}^{3} \boldsymbol{y}
$$

In the approximation of a source with low speed, one has:

$$
T^{00}=\rho c^{2} ; T^{0 i}=c \rho u^{i} ; T^{i j}=\rho u^{i} u^{j}
$$

And for a stationary solution, one has:

$$
\bar{h}^{\mu v}(\boldsymbol{x})=-\frac{4 G}{c^{4}} \int \frac{T^{\mu v}(\boldsymbol{y})}{|\boldsymbol{x}-\boldsymbol{y}|} \mathrm{d}^{3} \boldsymbol{y}
$$

At this step, by proximity with electromagnetism, one traditionally defines a scalar potential $\varphi$ and a vector potential $H^{i}$. There are in the literature several definitions as in [4] for the vector potential $H^{i}$. In our study, we are going to define:

$$
\bar{h}^{00}=\frac{4 \varphi}{c^{2}} ; \bar{h}^{0 i}=\frac{4 H^{i}}{c} ; \bar{h}^{i j}=0
$$

With gravitational scalar potential $\varphi$ and gravitational vector potential $H^{i}$ :

$$
\begin{gathered}
\varphi(\boldsymbol{x}) \equiv-G \int \frac{\rho(\boldsymbol{y})}{|\boldsymbol{x}-\boldsymbol{y}|} \mathrm{d}^{3} \boldsymbol{y} \\
H^{i}(\boldsymbol{x}) \equiv-\frac{G}{c^{2}} \int \frac{\rho(\boldsymbol{y}) u^{i}(\boldsymbol{y})}{|\boldsymbol{x}-\boldsymbol{y}|} \mathrm{d}^{3} \boldsymbol{y}=-K^{-1} \int \frac{\rho(\boldsymbol{y}) u^{i}(\boldsymbol{y})}{|\boldsymbol{x}-\boldsymbol{y}|} \mathrm{d}^{3} \boldsymbol{y}
\end{gathered}
$$

With $K$ a new constant defined by:

$$
G K=c^{2}
$$

This definition gives $K^{-1} \sim 7.4 \times 10^{-28}$ very small compare to $G$.

The field Equation (1) can be then written (Poisson equations):

$$
\Delta \varphi=4 \pi G \rho ; \Delta H^{i}=\frac{4 \pi G}{c^{2}} \rho u^{i}=4 \pi K^{-1} \rho u^{i}
$$

With the following definitions of $\boldsymbol{g}$ (gravity field) and $\boldsymbol{k}$ (gravitic field), those relations can be obtained from the following equations (also called gravitomagnetism): 


$$
\begin{gathered}
\boldsymbol{g}=-\operatorname{grad} \varphi ; \boldsymbol{k}=\boldsymbol{r o t} \boldsymbol{H} \\
\operatorname{rotg}=0 ; \operatorname{divk}=0 \\
\operatorname{divg}=-4 \pi G \rho ; \boldsymbol{r o t k}=-4 \pi K^{-1} \boldsymbol{j}_{p}
\end{gathered}
$$

With the Equation (2), one has:

$$
h^{00}=h^{11}=h^{22}=h^{33}=\frac{2 \varphi}{c^{2}} ; h^{0 i}=\frac{4 H^{i}}{c} ; h^{i j}=0
$$

The equations of geodesics in the linear approximation give:

$$
\frac{\mathrm{d}^{2} x^{i}}{\mathrm{~d} t^{2}} \sim-\frac{1}{2} c^{2} \delta^{i j} \partial_{j} h_{00}-c \delta^{i k}\left(\partial_{k} h_{0 j}-\partial_{j} h_{0 k}\right) v^{j}
$$

It then leads to the movement equations:

$$
\frac{\mathrm{d}^{2} \boldsymbol{x}}{\mathrm{d} t^{2}} \sim-\operatorname{grad} \varphi+4 \boldsymbol{v} \wedge(\boldsymbol{r o t h})=\boldsymbol{g}+4 \boldsymbol{v} \wedge \boldsymbol{k}
$$

From relation (11), one deduces the metric in a quasi flat space:

$$
\mathrm{d} s^{2}=\left(1+\frac{2 \varphi}{c^{2}}\right) c^{2} \mathrm{~d} t^{2}+\frac{8 H_{i}}{c} c \mathrm{~d} t \mathrm{~d} x^{i}-\left(1-\frac{2 \varphi}{c^{2}}\right) \sum\left(\mathrm{d} x^{i}\right)^{2}
$$

In a quasi-Minkowski space, one has:

$$
H_{i} \mathrm{~d} x^{i}=-\delta_{i j} H^{j} \mathrm{~d} x^{i}=-\boldsymbol{H} \cdot \mathrm{d} \boldsymbol{x}
$$

We retrieve the known expression [3] with our definition of $H_{i}$ :

$$
\mathrm{d} s^{2}=\left(1+\frac{2 \varphi}{c^{2}}\right) c^{2} \mathrm{~d} t^{2}-\frac{8 \boldsymbol{H} \cdot \mathrm{d} \boldsymbol{x}}{c} c \mathrm{~d} t-\left(1-\frac{2 \varphi}{c^{2}}\right) \sum\left(\mathrm{d} x^{i}\right)^{2}
$$

Remark: The interest of our notation (compare to the traditional notation of gravitomagnetism) is that the field equations are strictly equivalent to Maxwell idealization (in particular the speed of the gravitational wave obtained from these equations is the light celerity). Only the movement equations are different with the factor " 4 ". But of course, all the results of our study could be obtained in the traditional notation of gravitomagnetism with the relation $\boldsymbol{k}=\frac{\boldsymbol{B}_{g}}{4}$.

\subsection{From Linearized General Relativity to Gravitational Redshift}

In our approximation, we are going to seek for a relation of the gravitational potential that contain the gravitic component in addition to the gravity's term. It could be seen as a correction of the traditional gravitational potential that take in account only the gravity field. By this way (in the linearized approximation), others relations in which the gravitational potential intervenes could be adapted to take in account the gravitic field.

From the previous relation (16), one can write:

$$
\begin{gathered}
\mathrm{d} s^{2}=\left(1+\frac{2 \varphi}{c^{2}}-\frac{8 \boldsymbol{H} \cdot \mathrm{d} \boldsymbol{x}}{c^{2} \mathrm{~d} t}\right) c^{2} \mathrm{~d} t^{2}-\left(1-\frac{2 \varphi}{c^{2}}\right) \sum\left(\mathrm{d} x^{i}\right)^{2} \\
\mathrm{~d} s^{2}=\left(1+\frac{2(\varphi-4 \boldsymbol{H} \cdot(\mathrm{d} \boldsymbol{x} / \mathrm{d} t))}{c^{2}}\right) c^{2} \mathrm{~d} t^{2}-\left(1-\frac{2 \varphi}{c^{2}}\right) \sum\left(\mathrm{d} x^{i}\right)^{2}
\end{gathered}
$$


One can then define, in this approximation, a gravitational potential that could be qualified as "corrected" in the sense that the gravitic term is added to the traditional gravitational potential as a corrected term (with the velocity of the test particle, $\boldsymbol{v}=\mathrm{d} \boldsymbol{x} / \mathrm{d} t)$ :

$$
\varphi_{\text {CORR }}=\varphi-4 \boldsymbol{H} \cdot \boldsymbol{v}=\varphi+\varphi_{K 1}
$$

We can then apply these relations for the gravitational redshift:

$$
1+z=\left(\frac{1-\frac{2}{c^{2}} \varphi_{\text {CORR }, E}}{1-\frac{2}{c^{2}} \varphi_{\text {CORR }, R}}\right)^{1 / 2} \sim\left(\frac{1-\frac{2}{c^{2}}\left(\varphi_{E}+\varphi_{K 1, E}\right)}{1-\frac{2}{c^{2}}\left(\varphi_{R}+\varphi_{K 1, R}\right)}\right)^{1 / 2}
$$

\section{Gravitic Field and Gravitational Redshift at the Center of Our Galaxy}

\subsection{Simplified Relation for the Gravitational Redshift}

With $\varphi=-\frac{G M}{r}$ and $\|\boldsymbol{H}\| \sim \frac{K_{1}}{r}$ as in the approximation of [1], i.e. galaxy as a punctual mass distribution, one has (with $\theta_{\boldsymbol{H}, \boldsymbol{v}}$ the angle between $\boldsymbol{H}$ and $v)$ :

$$
\varphi_{\text {CORR }}=\varphi+\varphi_{K 1}=-\frac{G M}{r}-4 \frac{K_{1}}{r} v \cos \theta_{H, v}
$$

In our case of SO-2, one can write the contribution of each object (SO-2 and $\left.\operatorname{SgrA}^{\star}\right)$ :

$$
\begin{aligned}
& \varphi_{\text {CORR }, E}=\varphi_{S g r A^{*}}+\varphi_{K 1-S g r A^{*}}+\varphi_{S O-2}+\varphi_{K 1-S O-2} \\
& \varphi_{C O R R, R}=\varphi_{\text {Sun }}+\varphi_{K 1-S u n}+\varphi_{\text {Terre }}+\varphi_{K 1-\text { Terre }}
\end{aligned}
$$

Concretely it gives for the gravity potentials with the radius of SO-2 and distance to $\operatorname{SgrA}^{*}\left(r_{\mathrm{SO}-2} \sim 7 \times 7 \times 10^{8} \mathrm{~m}\right.$ and $\left.r_{\mathrm{SO}-2 \leftrightarrow S g r A^{*}} \sim 2 \times 10^{13} \mathrm{~m}\right)$ and the mass of SO-2 and $\mathrm{SgrA}^{*}\left(M_{\mathrm{SO}-2} \sim 13 \times 2 \times 10^{30} \mathrm{~kg}\right.$ and

$$
M_{S g r A^{*}} \sim 4 \times 10^{6} \times 2 \times 10^{30} \mathrm{~kg} \text { ) [5] [6]: }
$$

$$
\begin{aligned}
& \varphi_{S O-2}=-\frac{G M_{S O-2}}{r_{S O-2}} \sim-6 \times 10^{-11} \times \frac{13 \times 2 \times 10^{30}}{7 \times 7 \times 10^{8}} \sim-3 \times 10^{11} \\
& \varphi_{S g r A^{*}}=-\frac{G M_{S g r A^{*}}}{r_{S O-2 \leftrightarrow S g r A^{*}}} \sim-6 \times 10^{-11} \times \frac{4 \times 10^{6} \times 2 \times 10^{30}}{2 \times 10^{13}} \sim-2 \times 10^{13} \\
& \varphi_{\text {Terre }}=-\frac{G M_{\text {Terre }}}{r_{\text {Terre }}} \sim-6 \times 10^{-11} \times \frac{6 \times 10^{24}}{6 \times 10^{6}} \sim-6 \times 10^{7} \\
& \varphi_{\text {Sun }}=-\frac{G M_{\text {Sun }}}{r_{\text {Terre-Sun }}} \sim-6 \times 10^{-11} \times \frac{2 \times 10^{30}}{10^{11}} \sim-10^{9}
\end{aligned}
$$

That is:

$$
\begin{aligned}
& \frac{2}{c^{2}} \varphi_{S O-2} \sim-7 \times 10^{-6} \ll 1 \\
& \frac{2}{c^{2}} \varphi_{S g r A^{*}} \sim-5 \times 10^{-4} \ll 1
\end{aligned}
$$




$$
\begin{aligned}
& \frac{2}{c^{2}} \varphi_{\text {Terre }} \sim-10^{-9} \ll 1 \\
& \frac{2}{c^{2}} \varphi_{\text {Sun }} \sim-3 \times 10^{-8} \ll 1
\end{aligned}
$$

The gravity term of SgrA* is the main term. The others can be neglected. Or the gravitic terms, even if we don't know the value of the gravitic potentials $\varphi_{K 1}$ (it is the goal of the next section), we can expect that they will be less than 1.

In our case of SO-2, this relation can be then simplified $\left(\frac{2}{c^{2}} \varphi_{C O R R, E} \ll 1\right.$ and $\left.\frac{2}{c^{2}} \varphi_{C O R R, R} \ll 1\right)$ :

$$
\begin{aligned}
1+z & =\left(\frac{1-\frac{2}{c^{2}} \varphi_{C O R R, E}}{1-\frac{2}{c^{2}} \varphi_{C O R R, R}}\right)^{1 / 2} \sim\left(1-\frac{1}{c^{2}} \varphi_{C O R R, E}\right)\left(1+\frac{1}{c^{2}} \varphi_{C O R R, R}\right) \\
& \sim 1-\frac{1}{c^{2}} \varphi_{C O R R, E}+\frac{1}{c^{2}} \varphi_{C O R R, R}
\end{aligned}
$$

One can also expect that the gravitic terms $\left(\varphi_{K 1}\right)$ will follow the same order of classification than the gravity terms. The relation (25) can then be written (with (22)):

$$
z \sim-\frac{1}{c^{2}} \varphi_{C O R R, E}+\frac{1}{c^{2}} \varphi_{C O R R, R} \sim-\frac{1}{c^{2}} \varphi_{S g r A^{*}}-\frac{1}{c^{2}} \varphi_{K 1-S g r A^{*}}=z_{G}+z_{H}
$$

We are now going to study what could be the value of $\varphi_{K 1-S g r A^{*}}$ and $z_{H}$.

\subsection{Possible Values for the Gravitational Redshift}

\subsubsection{Comparison of $\varphi_{\text {CORR }}$ and $\varphi$ at the Scale of Our Galaxy (at Its Ends)}

For our galaxy we have $M \sim 2 \times 10^{42}$. It gives $G M \sim 10^{32}$. At the ends of the galaxy $v \sim 2 \times 10^{5}$ and from [1], in the galaxies we have the order of magnitude $K_{1} \sim 10^{24}$, it then gives $4 K_{1} v \sim 10^{30}$. At the better case for $\varphi_{K 1}$ (with $\theta_{\boldsymbol{H}, \boldsymbol{v}}=0$ ), in our galaxy, one would have $\varphi_{K 1} \sim 10^{-2} \varphi$ and then $\varphi_{C O R R} \sim \varphi$ in agreement with the fact that the gravitic interaction is negligible at the scale of the galaxy (that's also the reason why the dark matter cannot be explained by the own gravitic field of the galaxies).

\subsubsection{Comparison of $\varphi_{\text {CORR }}$ and $\varphi$ in the Center of Our Galaxy (SO-2 Case)} The difficulties of this computation are that we don't know the value of $K_{1}$, so close to the center of the Galaxy, and we don't know the angle between the velocity's vector $\boldsymbol{v}$ of SO-2 and the vector $\boldsymbol{H}$. Let's make a first computation by applying a scale factor compare to the previous situation of the whole galaxy. The mass of the supermassive black hole at the center of our galaxy is $M \sim 8 \times 10^{36}$. For the whole galaxy $M \sim 2 \times 10^{42}$, one can then apply the same factor $\left(4 \times 10^{-6} \sim 8 \times 10^{36} / 2 \times 10^{42}\right)$ to $K_{1}$, it then gives $K_{1} \sim 4 \times 10^{18}$. If we take for the velocity at the periastron of SO-2 $v \sim 4 \times 10^{6} \mathrm{~m} \cdot \mathrm{s}^{-1}$, one has 


$$
\begin{aligned}
& \varphi_{S g r A^{*}} \propto G M \sim 5 \times 10^{26} \\
& \varphi_{K 1-S g r A^{*}} \propto 4 K_{1} v \sim 6 \times 10^{25}
\end{aligned}
$$

One can also note that in addition to the difficulty of knowing the value of $K_{1}$, there is also the difficulty of knowing the angle $\theta_{\boldsymbol{H}, \boldsymbol{v}}$. If $\left|\theta_{\boldsymbol{H}, \boldsymbol{v}}\right|<84^{\circ}$ one has $\cos \theta_{\boldsymbol{H}, \boldsymbol{v}}>10^{-1}$, if $84^{\circ}<\left|\theta_{\boldsymbol{H}, \boldsymbol{v}}\right|<89.4^{\circ}$ one has $10^{-1}>\cos \theta_{\boldsymbol{H}, \boldsymbol{v}}>10^{-2}$ and for angles nearer to $90^{\circ}$ the cosine will be smaller. The vector $\boldsymbol{H}$ is expected to be in the plane of the galaxy [1], tangent to circles centered on the galaxy center. At the periastron, the velocity seems close to the plane of the galaxy. It seems likely that $\boldsymbol{v}$ and $\boldsymbol{H}$ are not too close to the perpendicularity and then that $\cos \theta_{\boldsymbol{H}, \boldsymbol{v}}$ is not too small.

But the application of our scale factor on $K_{1}$ most likely decreases the true value. Our previous values are an approximation when we have a punctual mass (when the studied body is far from a spatially extended source of mass). When we apply a scale factor for our case of SO-2 (from the whole galaxy to the center of the galaxy), unfortunately physically we are in a situation where there are masses beyond SO-2 (where the studied body is embedded in the spatially extended source). The punctual approximation must be enhanced by taking into account the surrounding matter. Unlike the centripetal gravity vector, the gravitic vector (similar to the magnetic vector in electromagnetism) can stay more constant because it is not a centripetal force. As the gravity field, the gravitic field will be more diluted (compare to the punctual idealization) but the gravity field of this surrounding mass can in addition be partially neutralized by some mass that are in the opposite side of SgrA* from SO-2, an effect that doesn't happen for the gravitic field. Then, the effect of the surrounding matter would less modify the computation of the gravitic field. Consequently to take in account this correction, one could modify $K_{1}$. This then means that $K_{1}$ should be greater. And only one order of magnitude ( $K_{1} \sim 4 \times 10^{19}$ ) could be enough to be of the same magnitude than the gravity field at the periastron of SO-2:

$$
\begin{aligned}
& \varphi_{S g r A^{*}} \propto G M \sim 5 \times 10^{26} \\
& \varphi_{K 1-S g r A^{*}} \propto 4 K_{1} v \sim 6 \times 10^{26}
\end{aligned}
$$

Then in the case of the measure of the gravitational redshift at the periastron, a discrepancy with the expected value could be measured, the value of $K_{1}$ and the velocity of SO-2 would be enough to generate a gravitic component of the same magnitude than the expected gravitational redshift due to the only gravity field.

$$
\begin{aligned}
& z_{G} \sim \frac{G M}{r c^{2}} \sim \frac{6 \times 10^{-11} \times 8 \times 10^{36}}{2 \times 10^{13} \times 9 \times 10^{16}} \sim 3 \times 10^{-4} \\
& z_{H} \sim \frac{4 K_{1} v}{r c^{2}} \sim \frac{4 \times 10^{19} \times 4 \times 10^{6}}{2 \times 10^{13} \times 9 \times 10^{16}} \sim 10^{-4}
\end{aligned}
$$

One can note that, far of the periastron, for almost all along the orbit of 
SO-2, the velocity is of about $v \sim 10^{5} \mathrm{~m} \cdot \mathrm{s}^{-1} \quad$ (ten times less than at the periastron). The gravity field stays then the main component. The contribution of the gravitic field $\left(z_{H}\right)$ compare to the gravity field $\left(z_{G}\right)$ would be, at the best case (the best orientation of the vectors as mentioned before), of one order of magnitude lower all along the orbit of SO-2, far of the periastron:

$$
\begin{aligned}
& z_{G} \sim \frac{G M}{r c^{2}} \sim \frac{6 \times 10^{-11} \times 8 \times 10^{36}}{2 \times 10^{13} \times 9 \times 10^{16}} \sim 3 \times 10^{-4} \\
& z_{H} \sim \frac{4 K_{1} v}{r c^{2}} \sim 4 \frac{4 \times 10^{18} \times 4 \times 10^{6}}{2 \times 10^{13} \times 9 \times 10^{16}} \sim 4 \times 10^{-5}
\end{aligned}
$$

It is then not impossible to forecast a discrepancy on the measure of the gravitational redshift of SO-2 at its periastron and to be in agreement with the general relativity. This discrepancy would reveal larger gravitic fields than expected for large structures.

What would happen if the gravitic field was greater $\left(K_{1} \geq 4 \times 10^{20}\right)$. In this case, the gravitic field would be of the same magnitude than the gravity field all along the orbit of SO-2 where the velocity is around $10^{5} \mathrm{~m} \cdot \mathrm{s}^{-1}$ (and even the main interaction at the periastron where $v \sim 4 \times 10^{6} \mathrm{~m} \cdot \mathrm{s}^{-1}$ ).

But this means that the deduced mass $\left(M \sim 8 \times 10^{36}\right)$ would already take into account the gravitic field. The deduced mass would then not only represent the baryonic mass $M_{B a r}$ but:

$$
M \sim M_{B a r}+\frac{4}{G} K_{1} \cos \theta_{H, v}
$$

In this case, to observe a discrepancy in the gravitational redshift, a large difference between the minimal and the maximal velocity would be necessary because this "average mass", which depends on the velocity (by taking into account the non-negligible gravitic field), would sufficiently change to generate a measurable discrepancy. At the periastron the velocity of SO-2 is more than ten times the lowest velocity of its orbit. One can then expect to measure a discrepancy on the observed gravitational redshift of nearly ten times greater than the expected one. One could then observe at the maximum:

$$
z_{H} \sim 10 \frac{G M}{r c^{2}} \sim 4 \times 10^{-3}
$$

In the frame of general relativity, this last case would be the greater measurable discrepancy. A greater discrepancy would be more difficult to explain by the general relativity.

\section{Conclusions}

SO-2 will be soon at its periastron for which its velocity will be around $v \sim 4 \times 10^{6} \mathrm{~m} \cdot \mathrm{s}^{-1}$. The expected gravitational redshift at this position is around $z_{G} \sim 3 \times 10^{-4}$. From [1], the gravitic field at the Galaxy's center could generate in the best case a measurable discrepancy on this gravitational redshift. The value of this gravitic field at the Galaxy's center remains unknown, but depending on 
its value several cases with specific consequences appears as a result of this study. The discrepancy on the gravitational redshift at the periastron could be due to the gravitic component $\left(z_{H}\right)$ and could lead to several situations. The order is also indicated in term of $\mathcal{O}\left(\beta^{n}\right)$ effect (where $\beta$ is the pericenter velocity of SO-2 in light units for which the expected gravitational redshift $z_{G}$ is $\mathcal{O}\left(\beta^{2}\right)$ ):

For $z_{H} \ll 10^{-5}\left(\ll \mathcal{O}\left(\beta^{3}\right)\right)$ the gravitic component would likely be lower than the calculated one of [1] at the center of the galaxy. But it will not invalidate the explanation of dark matter for which the gravitic field of the clusters must be the source. It is the expected measure for the more accepted theoretical frame [7].

$z_{H} \sim 10^{-5}\left(\sim \mathcal{O}\left(\beta^{3}\right)\right)$ would mean that the gravitic component at the center of the galaxy is greater than expected but would be always less important than the gravity field. In this case, the expected gravitic field for large structure would have to be revised upwards.

$z_{H} \sim 10^{-4}\left(\sim \mathcal{O}\left(\beta^{2}\right)\right)$ would mean that the gravitic component at the center of the galaxy is greater than expected and could be of the same importance than the gravity field (depending on the velocity of the studied object). In this case, the gravitic field would have to be revised upwards (as the previous case) but also the calculated mass of large structure would have to be revised downwards, following the relation (31) as a first approximation.

$z_{H} \sim 10^{-3}\left(\sim \mathcal{O}\left(\beta^{1}\right)\right)$ would mean that the gravitic component at the center of the galaxy is greater than expected and would even be the main gravitational component. This case would be at the limit of what it could be explain in the frame of the general relativity with a very important gravitic field.

If the discrepancy is greater, it could be a failure of general relativity. But for the other cases, this discrepancy wouldn't be a failure of the general relativity but at the contrary, it could be well explained by the general relativity itself by a more important term of gravitic field than expected. Furthermore, the measure of such a discrepancy would be a first important point for the way of explanation of the dark matter. In [1] the dark matter is explained by a more important gravitic field than expected (in particular for the clusters).

\section{References}

[1] Le Corre, S. (2015) Dark Matter, a New Proof of the Predictive Power of General Relativity. arXiv:1503.07440 [physics.gen-ph]

[2] Adler, R.J. (2015) The Three-Fold Theoretical Basis of the Gravity Probe B Gyro Precession Calculation. Classical and Quantum Gravity, 32, Article ID: 224002 https://doi.org/10.1088/0264-9381/32/22/224002

[3] Hobson, M., et al. (2006) General Relativity. Cambridge University Press, Cambridge. https://doi.org/10.1017/CBO9780511790904

[4] Mashhoon, B. (2008) Gravitoelectromagnetism: A Brief Review. arXiv:gr-qc/0311030

[5] Schödel, R., et al. (2002) A Star in a 15.2-Year Orbit Around the Supermassive Black Hole at the Centre of the Milky Way. Nature, 419, 694-696.

https://doi.org/10.1038/nature01121 
[6] Gillessen S., et al. (2017) An Update on Monitoring Stellar Orbits in the Galactic Center. The Astrophysical Journal, 837, Article ID: 30.

https://doi.org/10.3847/1538-4357/aa5c41

[7] Angèlil, R., et al. (2010) Relativistic Redshift Effects and the Galactic-Center Stars. The Astrophysical Journal, 711, 157-163.

https://doi.org/10.1088/0004-637X/711/1/157 